\title{
It could only happen to a doctor-Haemophilus aphrophilus septicaemia complicated by a prevertebral infection after dental work
}

\author{
A Poullis, S R Gould, A G Lim
}

\begin{abstract}
A 53 year old man presented with severe neck pain and a flu-like illness; he had recently returned from Sri Lanka and had had dental treatment six days before illness onset. Blood culture showed infection by Haemophilus aphrophilus. Magnetic resonance imaging was performed and exploratory surgery undertaken. The prevertebral cervical fascia was inflamed but no abscess identified. He was treated with antibiotics and made an uneventful recovery.

(Postgrad Med f 2001;77:261-262)
\end{abstract}

Keywords: Haemophilus aphrophilus; prevertebral infection

Haematogenous spread of oral commensals after dental work is a well recognised problem that has even received tabloid attention. ${ }^{1}$ Haemophilus aphrophilus (an oral commensal), is only rarely a cause of vertebral osteomyelitis or paravertebral abscess. Ten previous cases have been described but only two occurred after dental work and one case involved the cervical spine.

\section{Case report}

A 53 year old consultant physician presented with a painful neck 10 days into a flu-like illness. Of note he had returned from Sri Lanka one month previously and had visited the periodontologist six days before the onset of the illness. The neck pain was severe, requiring morphine analgesia. It was thought to be due to a locked facet joint and was treated with cervical traction. Rigors developed during the traction prompting a screen for infection and urgent revision of the diagnosis.

Investigations showed a mild neutrophil leucocytosis (total white cell count $9.3 \times 10^{9} / 1$ with $90 \%$ neutrophils), raised inflammatory markers (erythrocyte sedimentation rate $50 \mathrm{~mm}$ in one hour, C reactive protein $40 \mathrm{mg} / \mathrm{l}$ ), normal biochemistry (urea, electrolytes, and liver function tests) and serial malaria films were negative. Blood cultures showed small Gram negative rods, which were subsequently identified as Haemophilus aphrophilus. Treatment with ciprofloxacin and amoxycillin was started.

Magnetic resonance imaging (MRI, with gadolinium enhancement) showed increased uptake in the epidural space with possible abscess formation in the cervical prevertebral fascia (fig 1). A neurosurgical opinion was sought and surgical exploration was recommended. At operation the prevertebral cervical

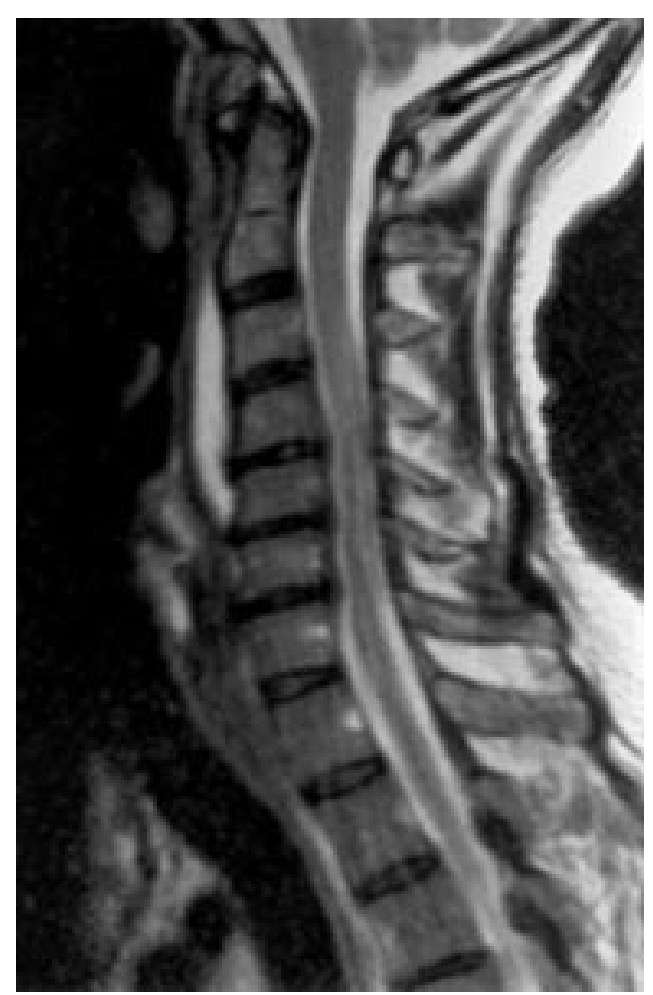

Figure $1 \quad T 1$ weighted MRI scan with contrast demonstrating increased uptake in the abnormal area.

fascia was inflamed but no abscess was identified. Biopsy specimens revealed granulation tissue but subsequent culture was sterile.

The patient was treated with intravenous amoxycillin and ciprofloxacin for 72 hours, at which stage gentamicin was added in because of a slow response. These were continued for three weeks followed by oral ciprofloxacin for two weeks. On this treatment the patient made a steady and uneventful recovery.

\section{Discussion}

$H$ aphrophilus is a slow growing, aerobic (capnophilic), Gram negative bacillus. Studies of asymptomatic adults have shown that haemophilus is ubiquitous, detected in $98.5 \%$ of supragingival and $96.2 \%$ of subgingival plaque samples. $H$ aphrophilus is more abundant in the deep subgingival area. ${ }^{2}$

Despite its ubiquitous presence in the oral cavity this is only the second case report in the English literature describing vertebral osteomyelitis or a paravertebral infection after a dental procedure. This case is the second case report in which $H$ aphrophilus has involved the cervical region (six other case reports involved the lumbar spine and three involved the thoracic spine). ${ }^{3}$ 
The diagnosis of vertebral osteomyelitis and spinal abscess is often difficult and therefore delayed this can lead to protracted illness, permanent neurological deficit, or death. ${ }^{4}$ For the treatment of spinal epidural abscess immediate surgical decompression has been advocated. ${ }^{4} \mathrm{~A}$ more conservative approach has been suggested if there is no neurological deficit or the deficit is mild and non-progressive and there is a clinical response with antibiotic treatment. ${ }^{5}$

$H$ aphrophilus is usually sensitive to $\beta$-lactam antibiotics, ciprofloxacin, and gentamicin; there are reports of resistance to $\beta$-lactam antibiotics in which case ciprofloxacin has been used. $^{6}$

As with all bone infections the key to diagnosis is to consider it. Severe unremitting bone pain, especially which does not settle with rest, may be non-mechanical in origin (tumour or infection). The history of rigors or "flu" should alert the doctor to the possibility of infection. A thorough history may reveal the likely cause, though obtaining a positive culture of the organism is most important for the treatment of the patient.

1 Dobson R. Dental dangers. Daily Mail 30 March 1999: 43. 2 Lijemark WF, Bloomquist CG, Uhl LA, et al. Distribution Infect Immun 1984;46:778-86.

\section{Learning points}

- In the presence of bone pain and rigors it is essential to think of and rule out osteomyelitis and or abscess formation and to consider potential sources of this.

- Preceding dental treatment is a potential source and should be inquired of in investigation of an unexplained pyrexia.

- Haemophilus aphrophilus, a common upper respiratory tract commensal, is a very rare cause of sepsis after dental work.

- Blood cultures are mandatory in all cases of unexplained fever.

- A label of "flu-like illness" may produce a false sense of diagnostic security.

3 Hung CC, Hsueh PR, Chen YC, et al. Haemophilus aphrophilus bacteraemia complicated with vertebral osteomyelitis and spinal epidural abscess in a patient with liver cirrhosis. F Infect 1997;35:304-8.

4 Danner RL, Hartman BJ. Update of spinal epidural abscess; 35 cases and review of the literature. Review of Infectious Diseases 1987;9:265-74.

5 Baker AS, Ojermann RG, Baker RA. To decompress or not to decompress - spinal epidural abscess. Clin Infect Dis 1992;15:28-9.

6 O'Driscoll JC, Keene GS, Weinbren MJ, et al. Haemophilus aphrophilus discitis and vertebral osteomyelitis. Scand $\mathcal{f}$ Infect Dis 1995;27:291-3. 